\title{
Seeking an expanded, multidimensional conceptual approach to health literacy and health disparities research
}

\author{
Robert A. Logan \\ National Library of Medicine (NLM), 8600 Rockville Pike, Bethesda, MD 20894, USA \\ E-mail:rlogan@mail.nih.gov
}

\begin{abstract}
This chapter compares the conceptual foundations of health literacy and health disparities. It details some of the conceptual differences between health literacy and health disparities and explains some similarities that suggest the need for increased research collaboration. The chapter is among the first to address the structural and social determinants of health together and explain that future research needs to assess their interaction.

Overall, the chapter creates a conceptual foundation as well as challenges future scholars/practitioners to take more multidimensional approaches to assess health's determinants. The chapter also attempts to demonstrate there is nothing more practical than good theory, or clear conceptual foundations.

The chapter is divided into four sections that address the following topics: three conceptual frameworks about the determinants of health; opportunities in health disparities and health literacy research; seeking an expanded, multidimensional conceptual approach to health literacy and health disparities research; as well as a conclusion. The chapter suggests there are vacuums in current research knowledge that need future attention - especially regarding the integration of health literacy and health disparities research.

Keywords: Health literacy, health disparities, social determinants of health, structural determinants of health
\end{abstract}

\section{Introduction}

This chapter attempts to provide a broader conceptual framework within an ongoing discussion about the integration of health literacy and health disparities research in the U.S. [67]. The chapter attempts to demonstrate there is nothing more practical than good theory, or clear conceptual foundations.

The chapter is written for future researchers and encourages them to set research agendas that will move the health literacy discipline forward. The chapter suggests there are gaps in current research knowledge that need future attention - especially regarding the integration of health literacy and health disparities research.

Although the chapter emphasizes health literacy research and developments in the U.S., some international health literacy work is included. The chapter's intent is not to review or summarize the health literacy and health disparities research literature, or provide a systematic review of research findings as these have been published recently $[17,25,26,49]$.

The chapter is divided into four additional sections that address the following topics: three conceptual frameworks about the structural and social determinants of health; opportunities in health disparities and health literacy research; seeking an expanded, multidimensional conceptual approach to health literacy and health disparities research; as well as a conclusion. 
Within the second section, 'three conceptual frameworks about the structural and social determinants of health,' three subtopics are addressed. They are: the structural determinants of health fostered within the health care delivery system (or health risks generated by patient care and the medical research process); some of the social determinants of health from a health disparities perspective; as well as some of the social and structural determinants of health from a health literacy perspective.

The second section also addresses the possible integration of health disparities and health literacy research as well as their conceptual differences.

The third section, 'opportunities in health disparities and health literacy research,' discusses some of the specific challenges that have been identified as gaps within the current health literacy and health disparities research.

The fourth section, 'seeking an expanded, multidimensional conceptual approach to health literacy and health disparities research,' addresses rationales to integrate the structural and social determinants of health in research and interventions. The conclusion summarizes the chapter's primary findings and makes suggestions about additional challenges for future researchers.

Before moving to these topics, the Calgary Charter's definition of health literacy provides the operational definition of health literacy used in this chapter. While Sorensen, Broucke, Fullam, Doyle, Pelikan, Slonska, and Brand note there is not a consensus definition of health literacy, they find diverse definitions of the term health literacy reflect perspectives from different disciplines, including clinical medicine and public health [95]. A recent perspective from the National Academy of Medicine (NAM) attests that it is difficult to define the term 'health literacy' because the field traverses an array of disciplines [88].

Until a new, consensus definition of health literacy is furnished by NAM or others, the Calgary Charter, proposed at an international meeting in 2012, probably provides the most comprehensive, multidisciplinary perspective. The Calgary Charter's definition of health literacy suggests:

- Health literacy allows the public and personnel working in all health-related contexts to find, understand, evaluate, communicate, and use information.

- Health literacy is the use of a wide range of skills that improve the ability of people to act on information in order to live healthier lives.

- These skills include reading, writing, listening, speaking, numeracy, and critical analysis, as well as communication and interaction skills [2].

A definition of health disparities is provided in the chapter's second section.

Also, while the Institute of Medicine's (IOM's) research prior to 2016 will be referred to in this chapter as the IOM, in 2016 the IOM changed its name to the National Academy of Medicine (NAM). The clarification is needed because the IOM/NAM commendably generate significant publications about health literacy, health disparities, and many of the areas are discussed in this chapter.

\section{Three conceptual frameworks: The structural and social determinants of health}

This section discusses three conceptual frameworks that expand the clinical triangle that often are associated with the etiology of illness and its prevention (genotype, phenotype, and lifestyle behaviors). The section suggests one of the contributions of 21 st century research is the increasing recognition that prevailing health disparities and poor health literacy across the U.S., as well as the health risks which occur within the U.S. health care delivery system, are significant determinants of individual and population health. 
This section partially reflects a salutogenic framework about health, which suggests individual and population health and illness are not limited to disease diagnosis, treatment, and prevention [64]. While a salutogenic conceptual framework suggests illness and health represent a multidimensional continuum, the research about the determinants of health cites specific biopsychosocial or structural areas where health risks are generated, or where risks might be lowered by diverse prevention or intervention efforts.

The first of the three conceptual frameworks discussed here focuses on a few, selected structural determinants of health that are fostered within the U.S. health care delivery system, or some activities within health care delivery, which impact health risks, health care outcomes, and health care utilization. The research in this area suggests higher or lower health risks can be generated as a result of specific events generated by the health care delivery system that may or may not be related to the routine diagnosis and treatment of diseases or conditions.

The second conceptual framework focuses on the health risks fostered by health disparities that influence health outcomes and the utilization of the health care delivery system. Within the U.S.-based health disparities literature, the aggregate toll of health disparities on the nation's health is suggested as exemplifying some of the social determinants of health [3,84].

The third conceptual framework focuses on the risks fostered by low health literacy that impact health outcomes and the utilization of the health care delivery system. Within the health literacy literature, the toll of low health literacy also is suggested as an illustration of a social determinant of health in the U.S. as well as other nations [82].

A National Academy of Medicine perspective recently noted while poor health literacy and ongoing health disparities each illustrate social determinants of health, the fields of health literacy and health disparities evolved independently $[67,81]$. The NAM perspective also suggests health literacy and health disparities research have not been collaborative or integrated, which suggest they need to be discussed as two different domains of health's social determinants [67].

To backup, one of the important 21 st century perspectives on health's underpinnings, as attested by the foci in the U.S. National Institutes of Health's Precision Medicine Initiative, suggests individual health and disease prevention are the byproducts of a triangular interaction among: phenotype (an individual's prior medical history and physical traits); genotype (an individual's genetic/genomic inheritance); and an individual's lifestyle (or behaviors known to impact health, such as smoking, exercise, substance abuse, and body mass index). One of the significant contributions of 21 st century health care may be an increasing capacity to assess how the interactions among genotype, phenotype, and individual behaviors impact the health and disease prevention of individuals and perhaps diverse demographic populations [11,33].

However, another significant contribution of 21 st century health research is an increasing understanding that illness and wellness also are impacted by: (a) the social, cultural, economic, and geographical environments in which one lives, (b) one's health literacy, and (c) the challenges, errors, and oversights that occur as a result of exposure to the health delivery system $[3,78,81,98]$.

Health literacy, health disparities, and 'quality chasm' research suggest the determinants of health should not be limited to the assessment of phenotype, genotype, and lifestyle patterns. Instead, health literacy, health disparities, and 'quality chasm' research suggest health and illness also are significantly influenced by diverse social and structural factors.

\subsection{Structural determinants of health}

Turning first to some structural determinants represented by the latent health risks generated by the health care delivery system, the IOM's 'quality chasm' reports suggest medical research processes, pa- 
tient care, and other byproducts of exposure from routine care can significantly impact individual and population health $[27,53,98]$.

In identifying one of specific health challenges derived from medical research processes, Unger, Cook, Tai, and Bleyer explain insufficient clinical trial participation impedes the development of the evidencebased foundation used to treat cancer as well as other diseases and conditions [97]. Unger, Cook, Tai, and Bleyer suggest insufficient clinical trial participation fosters long range health risks, which provides an example of an indirect, structurally-generated determinant of health [97].

Hartung, Zarin, Guise, McDonaugh, Paynter, and Helfand suggest the knowledge gaps between the published findings in refereed journals - and the more comprehensive datasets from which findings are derived - also may foster health risks (especially if medications and medical products are assessed on the basis of incomplete data) [50]. In turn, Hartung et al. infer the gaps between comprehensive data sets and published findings are a potentially detrimental byproduct of medical research processes, which represent an indirect, structurally-generated determinant of health [50].

More recently, Baker describes how the irreproducibility of some medical research poses foundational health risks because irreproducibility undercuts the reliability and validity of biomedical research, which means interventions may or may not evidence-based [23,42]. One recent estimate suggests 70 percent of lab experiments cannot be replicated by the original or other investigators [23]. The lack of reproducibility is perceived as a significant underlying challenge in biomedical and other research by 52 percent of respondents to a survey of biomedical research scientists [23]. Similar to the previous examples, the issues surrounding research reproducibility suggest routine medical research procedures can foster inadvertent but potentially detrimental health risks, which also may be characterized as a structural determinant of health.

Regarding some structural determinant issues generated within routine patient care, Demoly, Passalacqua, Pfaar, Sastre, and Wahn suggest poor patient adherence to provider directions is an enduring byproduct of provider-patient interaction [36]. Poor patient adherence is an acknowledged, underlying, significant barrier to better health outcomes throughout the health care delivery system as well as an enduring structural challenge, which is sometimes characterized an indirect, system-generated, structural determinant of health.

Similarly, the prior authorization of patient-provider care decisions (by insurers or others) provides an ongoing, significant source of patient and provider stress. Shah adds the prior authorization of patientprovider care decisions is ubiquitous within the U.S. health care system and carries with it significant health risks because it may undermine clinical efforts to strive for the highest standards of patient care [92]. The potential inability to pursue state-of-the art care is another indirect, system-generated structural determinant of health in the U.S.

In addition, recent findings suggest there may be 250,000 annual medical errors, or human and health care delivery system mistakes within U.S. clinical venues [68]. Makary and Daniel suggest the frequency of estimated medical errors may be the third highest cause of death in the U.S. In other words, self-generated clinical errors are linked to higher mortality rates than all existing diseases and conditions with the exceptions of heart disease and cancer [68]. Makary and Daniel's research updates a 1999 IOM report that projected between 49,000-98,000 annual deaths from medical errors. Yet, the actual number of clinical errors and deaths remain uncertain because there is not a diagnostic related group (or a systematic way) to classify them [61]. Regardless of the extent of deaths from clinical errors, HealthNewsReview.org suggests self-generated mistakes within the health care delivery system are underappreciated and represent a direct structural determinant of health that is self-generated within the health care delivery system [1]. 
Some other structural determinants of health that have been identified as embedded within the U.S. health care delivery system include significant differences in the costs of similar treatments among different medical care institutions (within the same city) as well as significant dissimilarities in medical outcomes for the same diagnoses among and between peer health care delivery centers throughout the U.S. $[45,53,71,76,79,96]$. The inconsistencies regarding medical costs often are seen as barriers to improved utilization of the health care delivery system and curbing medical costs $[43,45,71,79]$. Significant inconsistencies in patient outcomes for the same diagnosis (among and between peer medical centers) also are an acknowledged impediment to better overall health care in the U.S. [44]. While cost and care disparities partially are addressed by periodically revising the standards of care set by the Joint Commission, and the recommendations of evidence-based groups, such as the National Center for Quality Assurance, Agency for Health Research Quality, the National Preventive Services Task Force, and the Centers for Medicare \& Medicaid Services, the need for these efforts are a de facto acknowledgement of the role dissimilar costs and outcomes play in influencing the quality of health care in the U.S.

Overall, the degree that internally-generated issues associated with patient care, medical research, and clinical operations impact patient and population health suggest each are a contributor within a broad web of health's structural determinants. While the Kaiser Family Foundation notes each area represents a significant opportunity for research, the Foundation encourages researchers to differentiate between what they term 'health care disparities' (such as the structural determinants introduced above) and health disparities, which is suggested to be a different research field (reflecting a different perspective about health's determinants) [12].

The Kaiser Family Foundation operationally defines health care disparities as differences between groups in health insurance coverage, access to and use of care, quality of care, as well as some of the structural problems within the health care delivery system outlined above [12]. In contrast, the Kaiser Family Foundation suggests health disparities research focuses on a different conceptual dimension the higher burden of illness, injury, disability, or mortality experienced by one population group relative to others [12].

Briefly, a health disparities research perspective focuses on the socio-economic, cultural, demographic, and geographical variables associated with health risks and health outcomes. Although health disparities research does not challenge that structural health determinants can emerge within the health care delivery system or the aforementioned phenotype, genotype, and behavioral biopsychosocial triangle, health disparities research seeks to identify and assess some of the socially-derived factors that are associated with health outcomes.

\subsection{Health disparities}

More specifically, the field of health disparities suggests socially-derived demographic, socio-cultural, and environmental variables, such as gender, educational attainment, income, geography, public safety, housing and neighborhood quality, food security, and stressful living conditions, are statistically associated with comparative differences in health status across the U.S. $[3,25,49]$. The field of health disparities also can be extended to differences in health status among and between populations in other nations $[3,24,89]$.

While the identification of socially derived variables that impact health status may be historically tied to social discrimination or exclusion issues in the U.S. (such as race, ethnicity, religion), the intent of health disparities research is to evaluate the associations between diverse variables and health status and note comparative differences in population health. 
The National Institutes of Health operationally defines health disparities as the "difference in the incidence, prevalence, mortality, and burden of disease and other adverse health conditions that exist among specific population groups in the United States" [30]. The NIH definition seems to partially meet Braveman and Gruskin's criteria that a health disparities definition should focus on socially or structurally-derived patterns where a population group - already disadvantaged in terms of opportunity or resources - becomes increasingly more vulnerable to illness [28]. Adler adds the latter differences distinguish the field of health disparities from a focus on other population health inequalities, such as the superior health of young adults compared to senior citizens [16]. Adler infers the priority in health disparities research and practice should be more socially-generated, systematic, avoidable, and preventable population differences [16]. Interestingly, this important distinction may be partially buried in an operational definition of health equity as an ideal where people have the same and equal opportunities in order to reach their full health potential [4].

To backup, while the terms health equity, health disparities, and health care disparities are contemporary, the recognition that poverty, unemployment, deficient public safety, poor environmental conditions and diminished educational opportunities adversely impact a neighborhood's quality of life and health outcomes (compared to wealthier areas) is a leitmotif in some celebrated 19th and 20th century literature and films. While Sir Charles Chaplin's films and Charles Dickens' books sometimes end happily (based on a twist of fate that transforms the life of a vulnerable youth or person), their characters depict an anomie, angst, struggle, and stress (and a significant vulnerability) to remain healthy, safe, and sane compared to better opportunities in wealthier areas across town.

It is difficult to read Dickens or watch the Little Tramp without reaching an emotional appreciation of the sharp contrasts in health and safety and other aspects of life when comparing the lives of adults and children who live in vulnerable versus upscale neighborhoods. Dickens' novels and Chaplin's films also frequently convey an implicit understanding that while some social inequalities are inevitable, social indifferences about preventable disparities are reprehensible.

Fittingly, the specific focus of contemporary health disparities research is to empirically assess the extent of the sociological, demographic, geographic, and environmental associations in the U.S. with health status indicators and provide an unprecedented, evidence-based foundation for social interventions (presumably intended to improve the quality of life and health within nested vulnerable populations). While health disparities research is diverse and was recently summarized by Barr and Halvorson, four types of health disparities research findings are introduced here [25,49].

First, most health disparities research findings identify the demographic, socio-economic, and environmental variables that may impact health outcomes. Some of the frequently assessed independent (or predictor/control) variables that are associated with health outcomes include: race; ethnicity; gender; sexual orientation; age; disability; socio-economic status (SES); education; and geographic location. A second type of health disparities findings sacrifices some methodological range to provide more empirically robust (and generalizable) associations among fewer demographic predictor variables and health outcomes.

A third type of health disparities research findings assess if some socially-derived variables have a moderating effect on the associations among sociological, demographic, geographic, and environmental predictors with health outcomes. The latter is an important contribution because it identifies SES variables that potentially improve the health of vulnerable populations - and provides some evidence-based strategies that can be used in community-based interventions.

A fourth type of research findings are conceptually more multidimensional and identify how the health of vulnerable populations is impacted by interactions among socially-derived as well as structural vari- 
ables. Within the latter type of findings, socially-derived health determinants (regardless if they are sociological, demographic, geographic, or environmental) are seen as working in conjunction with some of the structural health determinants described earlier in this section. In turn, these findings suggest health outcomes are associated with diverse social and structural health variables that may be more intermediate than primary influences. Overall, the fourth type of findings suggest future health disparities research should strive to be more multidimensional, and include variables with an emerging evidencebase of health efficacy, such as health literacy.

Each of these four types of research findings is introduced below. Some individual studies fit within more than one of the aforementioned categories, which suggests some of the categories are not mutually exclusive. The first type of health disparities research seems to have many more examples in the literature than the other three. The review is followed by a brief discussion of health disparities interventions and the lack of integration of health literacy within health disparities research.

First, most health disparities research findings identify a range of sociological, demographic, geographic and environmental variables (often as independent variables) that are associated with significant differences in comparative health status or outcomes (often the dependent variables) across segments of the U.S. population. These research findings may identify one or more independent, or predictor/control variables and assess their impact on one or more types of health outcomes (or dependent measures).

For example, Nakaya and Dorling found two demographic variables (income and geography) were associated with one health outcome (mortality rates) [72]. In an overview of extant health disparities research, the U.S. Centers for Disease Control and Prevention (CDC) reported race, ethnicity, sex, sexual orientation, age, disability, socio-economic status (SES), and geographic location - both as singular or combined independent variables - were associated with single as well as diverse health outcomes [3]. Some of the diverse acute health outcomes identified by the CDC include: colorectal cancer; heart disease; stroke; HIV infection; preterm births; tuberculosis; and health related quality of life [3]. Some of the diverse chronic health outcomes identified by the CDC include: obesity; diabetes; and hypertension [3]. Some of the diverse behavioral health outcomes identified by the CDC include: cigarette smoking; binge drinking; suicide; as well as fatal and non-fatal work related injuries [3].

Barr also found SES, a multifaceted demographic variable, was associated with diverse health outcomes, including lung cancer, other cancers, coronary heart disease, other cardiovascular disease, stroke, chronic bronchitis and other respiratory disease, as well as gastrointestinal disease [25].

The CDC adds other research suggests a community's environment (e.g. access to green space, or local air and/or water quality) similarly is associated with skewed health outcomes among vulnerable populations [3]. In an example of research using an independent variable derived from sociological measures, researchers assessed if the use of community coalitions (within health disparities reduction campaigns) impacted desired health outcomes [20]. In a somewhat rare systematic review of health disparities research findings, a Cochrane review found common data elements, operational definitions, and measures to assess community coalitions were missing, which made it challenging to assess overall efficacy [20].

Most important, the CDC has reported there is an evidence-base to confirm hypotheses that an array of sociological, demographic, geographic, and environmental variables are associated with diverse, skewed health outcomes (that adversely impact vulnerable populations). However, many of the latter findings are derived from studies where comprehensive independent (or predictor) variables are not assessed simultaneously and may not be compared [3].

In examples where clusters of independent variables are evaluated simultaneously, Currie and Schwandt note health disparities researchers have assessed if one health outcome (mortality rates) is im- 
pacted by SES (based on educational attainment, geographic area, and income levels) combined with demographic variables (based on race and ethnicity) singularly, or in aggregate [35]. Currie and Schwandt conclude: 'Some studies investigating mortality across educational groups and geographic areas argue not only that inequality in life expectancy is widening, but that overall life expectancy is actually falling among the most disadvantaged groups' [35, p. 708]. Barr adds some health disparities research assesses the associations among a range of demographic and sociological variables (e.g. ethnicity, low position within a social hierarchy, low social capital, and exposure to discrimination) - with several, dependent health status measures (such as the formation of high individual stress, the subsequent development of injuries to tissues and organs, and significantly increased risks of illness and death) [25, p. 140].

A second type of health disparities findings sacrifices some methodological range in order to assess more empirically robust (and generalizable) associations among fewer demographic predictor variables and health outcomes. For example, Chetty, Stepner, Abraham, Lin, Scuden, Turner, Bergeron, \& Cutler recently found two demographic predictor variables (income and geography) are robustly associated with one health outcome (life expectancy rates in the U.S.) [32]. Chetty et al. suggest the top one percent in income (who live in wealthier communities) live 15 years longer than the poorest one percent of Americans who live in impoverished areas [32]. Chetty et al. add the wealthiest Americans (who live in wealthier communities with more social, environmental, and cultural amenities) gained more than three years of life expectancy during the first 14 years of the 21 st century - while no other demographic group (based on income and neighborhood clusters) experienced similar results [32]. From 2001-2014, changes in life expectancy ranged from gains of more than four years to loses of more than two years comparing wealthier to lower income neighborhoods [32].

The Chetty et al. study has been touted as a landmark because its findings are based on a quasi-universe rather than a sample of American adults, which means the results may be generalizable to the U.S. adult population. In an editorial accompanying the results, Woolf and Purnell suggest the results are a 'call to arms' for health care professionals to collaborate to improve population health [101]. Yet, in contrast to the first type of health disparities research, the Chetty study's findings are based on a comparatively narrow range of demographic and related independent variables as well as health outcomes. While the study's focus provides robust statistical power, its comparatively limited range suggests a distinctive type of health disparities research as well as a calculated tradeoff [32].

A third type of health disparities research assesses if some socially derived demographic variables have a moderating effect on the associations among sociological, demographic, geographical, environmental predictors and health outcomes. Returning to the Chetty et al. study, the findings suggest the impact of income and geography as predictive variables in skewed life expectancy can be moderated by community-based efforts that impact specific health behaviors, such as curbing smoking, improving weight control, and fostering exercise [32]. In a review of meta-analyses, Pascoe and Richman found perceived discrimination and resulting stress may be moderated by emotion-focused strategies, changes in healthy eating habits, increased social support, and group identification [83]. In turn, the identification of moderating variables are helpful to future health disparities researchers because they identify evidence-based SES, or other factors, that might contribute to more successful community-based interventions for vulnerable communities. Simultaneously, the identification of moderating effects yield distinctive insights that are not necessarily raised in other health disparities research findings.

A fourth type of health disparities findings identifies how the health of vulnerable populations is impacted by interactions among socially-derived as well as structural variables. In other words, these studies evaluate how sociological, demographic, geographical, or environmental variables interact with structural health determinants. 
For example, Currie and Schwandt recently found life expectancy disparities may be influenced by (and associated with) recent improvements in access to health insurance among young Americans [35]. (The access to health insurance among young Americans provides an example of the integration of a structural variable into health disparities research.) Currie and Schwandt suggest if declining differences in insurance access are projected to a future adult population, comparative mortality rates (a key dependent variable measure of health disparities) may become less significant [35].

Similarly, the Chetty et al. study suggests socially-derived SES variables work in conjunction with clinical variables (in this case smoking, exercise, weight control) to impact individual and community health [32].

Overall, these examples (among others) suggest population health outcomes sometimes are associated with multidimensional social and structural health variables - and all may work in conjunction with each other to impact health outcomes. This type of research provides an alternative to health disparities findings where specified independent (or primary, control, or predictor) variables (based on social, environmental, and other predictors) are seen to more directly impact health outcomes.

In addition to the interaction among variables, the fourth type of health disparities research findings suggest extant variables (sociological, demographic, geographical, environmental and structural) may be an intermediate compared to a direct or primary influence on health outcomes.

In short, the fourth type of health disparities findings provides an alternative, conceptual framework to understand the dynamics of health disparities. While some research may narrow the sociological, demographic, geographical, or environmental variables that predict health outcomes, the fourth research genre suggests the range of social and structural variables should be expanded - and researchers should expect most variables to have an intermediate (as opposed to a primary) impact on health outcomes.

To backup momentarily, a variable is classified as 'intermediate' when it results in empirical variation within both a study's dependent and independent research variables. In the Chetty study, the intermediate variables (smoking, exercise, weight control) interact and influence both the dependent variable (mortality) and the independent variables (income/geography SES variables) [32]. Hence, an intermediate association among and between variables in health disparities research suggests there is a degree of variation as well as a conceptual interdependence in modeling how health outcomes interact with social and structural determinants of health.

The finding that both social and structural variables may be interdependent as well as intermediate variables suggests two of the pressing challenges in health disparities research are to holistically conceptualize and assess the empirical associations among social and structural health determinants. Overall, the research provides a foundation to suggest conceptual advances in health disparities research might be enhanced by the addition of evidence-based social and structural determinants of health. In turn, this suggests future health disparities research might strive to be more multidimensional and include variables where there is an emerging evidence-base of health efficacy, such as health literacy.

Before we turn to an overview of health literacy, current initiatives such as the Robert Wood Johnson Foundation's (RWJ) community-based interventions, demonstrate how health disparities research is applied within community-based settings [5]. The RWJ intervention includes diverse initiatives designed to address community education, provide employment, augment food security, build or expand public parks, as well as enable access to better health care, and programs to modify specific health behaviors, such as smoking, obesity, and exercise [5]. While RWJ programs to address community education, provide employment, augment food security, build or expand public parks, and similar efforts are grounded in social (or sociological, demographic, geographical, environmental) determinants of population health, 
efforts to improve access to health care, and address smoking, weight control, and exercise are grounded in structural and clinical determinants of population health [5].

While a National Academy of Medicine perspective recently identified the combined RWJ interventions as an example of applied, comprehensive health disparities research, the NAM perspective added the RWJ initiative additionally illustrates the missing opportunities for collaboration between health disparities and health literacy researchers [67]. The NAM perspective reports the RWJ interventions do not include health literacy initiatives despite evidence that health literacy interventions have an intermediate impact on health outcomes and the utilization of the health care delivery system (or represent an evidence-based social determinant of health) [67].

On the other hand, the NAM perspective acknowledges the disciplinary gap is reciprocal - health literacy research often fails to include health disparities research in creating health literacy conceptual frameworks, methodological approaches, and initiatives [67].

While the NAM perspective notes more research collaboration should be a priority, the perspective adds the lack of cooperation probably is derived from different research funding streams as well as some comparative differences in health literacy's genesis and development [67].

Health literacy's genesis and expansion will be reviewed in the next subsection. Since the author recently reviewed the development of health literacy research, the discussion within the following subsection is somewhat abbreviated [66].

\subsection{Health literacy}

This subsection is divided into a discussion of health literacy's distinctive conceptual foundations and an overview of some major research findings is provided. We will explain how some early hypotheses about health literacy were confirmed by a national assessment of health literacy skills, which helped frame some of the current field's research approaches and its separation from health disparities research. The latter provides some additional reasons why health disparities and health literacy research have not been well integrated.

A recent systematic overview of health literacy's research findings suggests health literacy is a social determinant of health $[17,26]$. While the same systematic review notes health literacy research's future should feature an increased convergence of intermediate variables that include other social determinants of health (e.g. health disparities findings) and some structural determinants of population health, this section suggests the issue whether health literacy is a primary or intermediate variable is foundational to health literacy research's integration within related disciplines [17,26].

First, health literacy has become a self-contained area of study within the broader fields of adult literacy, health education, and health communication [54,58,82]. Health literacy's research interests also dovetail with other disciplines including: numeracy; consumer health informatics; cultural competence; eHealth, mHealth; patient activation; patient health self-management; health information seeking; shared health decision making; health prevention; adult literacy; risk assessment; mass media literacy; community-based health interventions; and the public understanding of science $[13,34,52,56,58]$. PubMed's topic-specific query page on health literacy (that provides a gateway to research published in major medical/public health research journals) encompasses many of the aforementioned disciplines and expands as relevant research surfaces from related sub-disciplines [14].

Second, health literacy research and practice are designed to help address an array of enduring clinical and health care delivery system consumer communication challenges such as the understandability of: medical consent forms; medical insurance forms; hospital signage; medical terminology; as well as drug 
labeling and instructions. Other enduring challenges include the clarity of hospital discharge instructions, and fostering more meaningful patient/provider interpersonal communication interactions. Health literacy research and practice also are associated with broader issues such as: patient and consumer education; patient enablement and empowerment; patient-centered clinical practices; the education and development of health care professionals; plain language; patient adherence to clinical instruction; health care cost containment; palliative care; efficient use of the health care delivery system; and issues such as precision medicine and the public understanding of medical policy initiatives, such as the U.S. Affordable Care Act [57,66,73].

Third, while there is extant evidence that health literacy is a social determinant of health, a recent systematic review suggests health literacy is an intermediate (rather than a primary) as well as a probable moderating variable that impact health outcomes and the utilization of the health care delivery system $[17,26]$. The implications of these findings for future research are discussed at the end of this subsection.

Although health disparities research focuses on the demographic, sociological, geographic, environmental, and structural variables that skew differences in health outcomes (among vulnerable populations), health literacy's raison d'etre remains the challenges most adults have (regardless of demographic, socio-economic, or geographical underpinnings) to understand medical terms, seek information, as well as the enduring frustrations that are byproducts of utilizing the health care delivery system $[54,56,66]$. Health literacy's foundation also includes the residual socio-economic, clinical, and public health impact of consumer frustration, misunderstanding, disinterest, disengagement, anxiety, and anomie in dealing with personal illness and clinical care $[54,56,66]$.

Other pressing reasons to improve the nation's health literacy (as well as address the impact of low health literacy on public health and medical care) are introduced within a recent executive order to create a health literacy partnership within a U.S. state [6]. The governor of Alabama's 2016 executive order explains almost nine out of ten Americans have difficulty understanding and actualizing the health information that is routinely available through the news media, advertising, commercial health products, and from health care facilities. The executive order notes adults with low health literacy are twice as likely as others to be hospitalized, are more likely to have chronic health issues, and are less likely to seek treatment. The executive order adds as health care costs rise, low health literacy is estimated to add as much as $\$ 238$ billion to an overburdened U.S. health care system [6].

While a health literacy leitmotif may not be prominent within Dickens' novels or Chaplin's films, prior to the use of the term 'health literacy' Nelkin as well as Gregory and Miller explained the public's understanding of health and medicine was a latent barrier (as well as a potential facilitator) to improve U.S. public health and medical care [48,74]. While some generalizable findings in the late 20th century suggested a dearth of knowledge about science among most Americans, the first empirically rigorous assessment of the public understanding of health and medicine did not occur until the National Assessment of Adult Literacy (NAAL) in 2003 [7,70]. In short, the extent of the public's understanding of health and medicine was not empirically exposed until the early 21 st century.

The NAAL, which remains the only generalizable evaluation of health literacy in the U.S., found 88 percent of Americans either had below basic, basic, or an intermediate understanding of basic medical terms and information. Only 12 percent of Americans were proficient in understanding medical terms or information $[7,63]$. Other international research findings suggest similar low levels of health literacy in diverse nations [56,63,90,95,99].

While most of the latter research provides demographic breakdowns, the findings are more generalizable to the full sample instead of demographic segments (or subpopulations) within many national and international health literacy assessments. 
Prior to the NAAL and similar findings, Leonard and Ceci Doak (health literacy's U.S. pioneers) hypothesized that an adult's age, income, educational attainment, and adult literacy levels may not predict his or her understanding of medicine, medical terms, as well as consumer interest in seeking information about health and medicine [6,38,40,41]. The Doaks' informal hypothesis also suggested that demographics might be less associated with high literacy proficiency than the health disparities literature might suggest [38-41]. In other words, the Doaks' hypothesis inferred health literacy's diffusion within subpopulations was somewhat inconsistent with the patterns suggested in health disparities research. While the Doaks noted that low health literacy was omnipresent within vulnerable populations, they added low health literacy was ubiquitous among all social classes and backgrounds [38-41]. Since the mixed findings in the NAAL and similar international studies suggest a suboptimal health literacy proficiency is consistent among undifferentiated large populations with more mixed results for subpopulations, health literacy's dispersion seems to be characterized by both its population similarities and differences. In short as the Doaks suggested, health literacy's population distribution has some similarities as well as important differences compared to the skew towards vulnerable populations found in health disparities research.

Prior to the NAAL, the Doaks also suggested health literacy was conceptually distinctive from adult literacy and each should be measured as separate constructs (rather than co-mingled or used interchangeably as surrogate measures) to assess the capacity of the public to understand health and medicine [3841].

In contemporary practice, adult literacy often is perceived as a combination of reading skills, document comprehension, writing skills, as well as functional abilities to understand pragmatic information, such as directions, instructions, numerical descriptions, and maps [54,77]. In contrast, health literacy focuses on a different set of abilities, skills, tasks, and conceptual underpinnings within clinical care, public health, health care management, patient/consumer, home, and other health information seeking contexts $[54,77]$. Nutbeam described health literacy skills within three categories: functional/technical skills (ability to read and understand numbers); interactive/social skills (listening, speaking); and critical thinking skills (the ability to integrate information within new life situations and challenges) [77]. The complexity of the latter additional skills suggests why adult literacy training sometimes is not perceived as surrogate for health literacy training among health educators and other practitioners.

In addition, the Doaks' suggestion that health literacy and adult literacy should be assessed as different research constructs (and not used interchangeably as surrogate measures) was operationalized within the NAAL's methodological approach - and partially bolstered by its findings. The NAAL findings suggested that health literacy, adult reading skills, as well as other literacy levels of U.S. adults were somewhat inconsistent $[7,63,99]$. The levels of health literacy proficiency (or the ability to understand medical terms and information) were somewhat differentiated from the other measures of adult reading skills $[7,63,99]$. So, besides a methodological separation of health and adult reading skills, the NAAL findings provided some initial evidence that the measurement of health literacy should be more grounded in measures tailored to assess health literacy than adult literacy. The latter distinction is noteworthy because adult literacy is significantly associated with educational attainment, which is a foundational demographic variable in health disparities research.

In recent years, the expansion of a literature that assesses health literacy instruments (quantitative, applied measures of health literacy) - and raises questions about which instrument to use - suggests a tacit consensus that health literacy is not a surrogate measure of adult literacy and suggests health literacy constructs should be utilized to measure the public understanding of health and medicine [66,85]. As a result, the separation of adult reading skills and health literacy into different constructs may have 
fostered a proliferation of 21 st century, self-contained health literacy measures as well as a de-emphasis on the utilization of health disparities measures and approaches in health literacy interventions $[56,85]$.

The separation of health literacy and adult literacy into self-contained research constructs also is conceptually important because it yields more insights about why some aspects of health disparities and health literacy research evolved separately in recent years.

Essentially, if health literacy and adult literacy are empirically independent constructs, this suggests adult literacy is not necessarily an integral component of an evidence-based health literacy conceptual framework. If adult literacy can be omitted, then, this justifies the possible exclusion of the demographic variables to which it is closely related, such as educational attainment, as well as some of the sociological, demographic, geographic, and environmental variables that are associated with both educational attainment as well as other disparate health outcomes. Hence, the exclusion of adult literacy and rejection of it as a surrogate measure of health literacy creates both conceptual as well as operationalized approaches that encourage researchers to adopt health literacy measures as self-contained independent variables. Conversely, if health literacy and adult literacy were analogous constructs, additional measures based on educational attainment (that are linked to variables used in health disparities research) might have been more valued and seen as part of an integrated, evidence-based, conceptual research framework.

Turning now to the reasons for the health care delivery system's 21 st century burst of interest in health literacy, the appeal of health literacy initiatives and research was accelerated by a convergence of elements including: the aforementioned population research that suggested widespread, low health literacy levels; health information/communication technological developments (such as eHealth and mHealth); the initial research findings about health literacy's desirable impact on patient and health administrative outcomes; the comparative advantages of health literacy as a tactic of clinical, organizational, and social intervention; as well as a self-perpetuating momentum derived from increasing government and private health care organizational interest $[62,65,66,75,82]$. Regarding the latter example, the U.S. Joint Commission's (the National Committee for Quality Assurance) inclusion of health literacy within their accreditation standards in 2011 reinforced that health literacy initiatives and interventions represented pragmatic strategies to address clinical care and broader administrative health related issues [8]. In the U.S., recent innovations in the Affordable Care Act, such as Patient Centered Medical Homes and Accountable Care Organizations, also encouraged a new range of programs and institutional accountability that recommended some health literacy initiatives and evaluation research $[59,60,82]$.

In addition, the comparative advantages of health literacy as a tactic of clinical, organizational, and social intervention was fostered by evidence that suggested therapeutic patient and public health outcomes might be generated by comparatively modest (e.g. less expensive) interventions designed to: boost patient and community understanding of health and specific conditions; foster more health selfmanagement skills; improve quality of patient interaction at key points of health care interaction (such as prior to hospital discharge); and generate health information seeking (often using new, less costly digital technologies). In turn, health literacy interventions seemed to provide a portfolio of pragmatic and affordable strategies for health care organizations to consider - especially when planning health care delivery outreach initiatives and interventions [66].

The progress in health literacy research was described in a landmark systematic review from the U.S. Agency for Healthcare Research and Quality (AHRQ) published in 2011 [17,26]. Among other important findings, the AHRQ systematic review initially found low health literacy was associated with some important health challenges, such as improved health status and reduced mortality rates $[17,26]$. 
Koh et al. and Bailey, Oramasionwu, and Wolf added some of the clinical benefits linked to health literacy interventions include: reduced mortality; improved patient adherence to medical instructions; and overall patient safety $[21,60]$. In a summary of research findings, the National Network of Libraries of Medicine noted health literacy interventions therapeutically assisted patients with cancer, diabetes, asthma, and hypertension [9].

In addition, the aforementioned AHRQ systematic review of health literacy research found an array of health care delivery challenges associated with low health literacy, such as: increased hospitalizations; more use of emergency care; and a reduced ability to understand both medication labels and health messages $[17,26]$. Koh et al. added some health literacy interventions were linked to improved utilization of the health care delivery system [60]. Koh et al. noted other specific health administrative benefits linked to health literacy interventions included: improved diabetes patient self-management skills; more use of preventive services; as well as a reduction in hospitalization and re-hospitalization rates (which in aggregate lower medical costs) [60].

In addition to noting some specific examples of health literacy's impact, the AHRQ systematic review suggested there is a sufficient evidence base to support the hypothesis that health literacy is a sociallyderived determinant of population health $[17,26]$.

Conversely, the evidence was more mixed whether health literacy (as an independent research construct) is a primary variable that influences outcomes, or whether health literacy is an intermediate variable that interacts with other social and structural determinants of health [17,26]. The issue is important to health literacy research's future because hypothetically, if health literacy is a primary, or robust, predictor of outcomes, then, this evidence justifies a continued separation of health literacy and health disparities research into self-contained research framing and methods. However, if health literacy is an intermediate variable that is interdependent with other socially-derived and structural determinants of health (as discussed earlier), this suggests a need for more integration of health literacy and health disparities research framing and methods - and moving away from conceiving health literacy and health disparities as separate research challenges and questions.

Some evidence that health literacy might be a primary variable was suggested by Baker's findings in 2006 [22]. At a conference sponsored by the U.S. Office of the Surgeon General, Baker reported health literacy (measured as an independent construct) was a robust predictor of patient health, such as mortality and self-reported health status, as well as a few health care administrative outcomes, such as hospitalization rates, even when compared to traditional demographic predictors of health outcomes [22]. Moreover, Baker suggested health literacy was a robust predictor of desirable patient and health administrative outcomes even after controlling for social-demographic variables, such as income and educational attainment [22].

The evidence that health literacy may be an intermediate (as well as a moderating) variable was suggested by the more recent and comprehensive AHRQ systematic review [17,26]. The AHRQ systematic review suggested health literacy should be conceptually modeled as one among other possible social and structural variables that interact to predict health outcomes, or impact the use of the health care delivery system $[17,26]$.

While acknowledging the current evidence is insufficient to provide a definitive position, the aforementioned NAM perspective recently suggested that health literacy is: a) a social determinant of health and b) probably is an intermediate and moderating variable that interacts with other social determinants of health (often represented by the variables used in health disparities research) as well as the structural variables discussed earlier [67]. 
Assuming the NAM perspective (and AHRQ systematic review) are reinforced by future research, it seems prudent to encourage researchers to seek an integration of social and structural determinants of population health in health literacy research rather than operationalize health literacy as a more singular, primary variable. Using the salutogenic conceptual foundation that was discussed at the start of this section, it also seems timely for research to take a multidimensional and multidisciplinary view when assessing social and structural determinants of population health in research planning, framing, methods, and implementation.

However, it also is important for future researchers to remain mindful that the larger topic of health literacy's integration or separation from health disparities research remains an important issue in advancing the research within both subdisciplines.

Finally, both health literacy and health disparities are challenged by an array of identified research gaps and methodological issues that undermine progress within both fields. Some of these research gaps and methodological issues research will be discussed in the next section. However, the intent is more to more to identify existing opportunities for researchers in both research areas rather than critique current shortcomings.

\section{Opportunities in health disparities and health literacy research}

While overviews of health disparities and health literacy research describe some of the diversity and vitality of research in both fields, some significant evidence gaps and resulting needs in each area also have been identified $[25,66,85]$. This section introduces some of current evidence gaps that have been acknowledged in health disparities and health literacy research respectively. The intent is to encourage researchers to interpret the suggested evidence gaps or challenges as opportunities to elevate the authority and credibility of both disciplines.

Turning first to health disparities research, an Institute of Medicine health indicators report suggested revising an array of measures that provide foundational structural and demographic health information [55]. The IOM report also suggests some current definitions and operationalization of variables within areas of structural and demographic health outcomes do not provide a foundation for high quality data that is needed in public and population health research, which includes health disparities [55]. For example, the IOM report provided and defined revised measures of health outcomes, such as: life expectancy at birth; infant mortality; life expectancy at age 65; injury related mortality; self-reported health status; unhealthy days physical and mental; chronic disease prevalence; and serious psychological distress [55]. The report provided and defined revised measures of common health outcomes, such as: smoking; physical activity; excessive drinking; nutrition; obesity; and condom use [55]. The report provided and defined revised measures of health system activities, such as: health care expenditures; insurance coverage; unmet medical, dental, and prescription drug needs; use of preventive services; childhood immunization; and preventable hospitalizations [55].

While the IOM health indicators report did not provide revised definitions of specific measures of the social determinants of health, the report emphasized future efforts were needed to create revised measures in both health disparities research and the larger field of population health [55]. Overall, the health indicators report strongly suggests the current measures of social and structural determinants of health are not optimal to provide needed insights about national trends especially for systematic reviews grounded in data aggregation and harmonization [55]. In turn, this suggests there are important challenges to improve basic research about population health and health disparities and the status quo of current research methods needs some reconsideration. 
Similarly, the aforementioned AHRQ's health literacy systematic review suggested health literacy research needs more conceptual consistency, more research about its underlying measures, and a new emphasis on best research practices to generate data sets that provide a better foundation for future research as well as systematic analyses [17,26].

Regarding health literacy's current conceptual inconsistencies, most attention within this area focuses on the proliferation of health literacy definitions and the current lack of a consensus definition. For example, in a landmark paper, Sorensen et al. outlined an array of health literacy definitions and noted how each reflected underlying differences in conceptual frameworks that included clinical practice versus public health and other perspectives [95].

While Sorensen et al. and Pleasant agreed that the subtle differences among health literacy definitions and underlying conceptual models suggested the field's conceptual diversity, they countered the lack of a consensus about health literacy's definition - coupled with diverse underlying conceptual models inadvertently challenge the field's long range scholarly credibility and gravitas [85,95].

Although the author adopted the Calgary Charter definition in this chapter's introduction, Pleasant adds the current inconsistencies among and between basic health literacy definitions can confuse researchers, and suggests the field lacks a sense of direction or scholarly leadership $[2,86]$.

A recent NAM perspective explains while it is difficult to reach a consensus definition of health literacy, the perspective outlines some of the underlying issues that need to be resolved in order to achieve a consensus definition [88].

To backup, one of health literacy's current research and conceptual gaps is to answer an essential question: what is health literacy and how should it be defined?

In addition to a missing consensus definition, Pleasant notes there is a pressing need for more research regarding the quantitative rigor of health literacy's underlying measures [85,86]. Pleasant and others explained many of the health literacy field's diverse definitions and conceptual models fostered the use of diverse and sometimes incompatible research instruments [51,85-87]. Haun et al. recently described the diversity of the 51 health literacy measures they identified as 'instrument proliferation' [51, p. 301]. Similar to the Agency for Healthcare Research and Quality's systematic review, Pleasant explained current diverse and often incompatible research instruments make it difficult to aggregate research findings as well as compare results that might yield more systematic insights about conceptual models and instruments [17,26,86].

Pleasant and McCormack, Haun, Sorensen, \& Valerio also found many health literacy quantitative research instruments have not been undergirded by basic research that demonstrates their psychometric rigor $[69,85,86]$. In a systematic review, Altin, Finke, Kautz-Freimuth, \& Stock recently found only 17 articles focused on the development and validation of 17 health literacy instruments [19]. Pleasant suggests the latter issues inhibit investigators' abilities to make informed choices about which health literacy instrument (and underlying conceptual model) are optimal to use $[51,85,86]$.

As a remedy, O'Neill, Goncalves, Ricci-Cabello, and Ziebland, Altin et al., Haun et al., McCormack et al., and Pleasant noted the need for conceptual transparency as well as more basic research and validation studies about health literacy's diverse instruments [19,51,69,80,85,86].

Soon after these critiques were published, healthliteracy.bu.edu was launched to provide a clearinghouse of health literacy measures as well as links to the extant validation research undergirding each measure. While healthliteracy.bu.edu is a direct response to providing more transparency about the rigor of the common health literacy instruments, the site's future success depends on the expansion of basic research regarding health literacy's research instruments. Full disclosure: the author was involved in the creation of healthlitercy.bu.edu. 
More broadly, Dewalt and Hink found while there is an abundance of health literacy initiatives, there is a comparative dearth of evidence-based assessments of health literacy interventions [37]. Abrams, Klass, and Dreyer found a similar dearth of evidence-based assessments of health literacy interventions especially within community-based interventions tailored to low literacy populations [15]. In aggregate, both findings suggest one of health literacy's most significant challenges is to increase the use of evidence-based research to assess the impact of clinical or community interventions.

The extant literature additionally identifies specific areas or topics within health care delivery and patient care where there is a dearth of health literacy evidence and scholarship. While the implication of much of this literature is health literacy cannot evolve as a field of study until these areas receive more scrutiny, each of the identified areas additionally opens an opportunity for future researchers.

For example, Koh et al. identified a range of research needs in health care administrative settings such as: how intra-organizational communication affects the processes and outcomes for health care organizations that seek to be more health literate; what interpersonal and/or organizational strategies help reduce the burden on individuals to coordinate their own medical care, and how health information systems best engage patients and caregivers by providing easily understandable, personalized medical record data as well as other clinical information [59].

Koh et al. added health administrators also need health literacy research about areas such as: how health literacy (in conjunction with health communication and health information technology) contribute to more shared decision making between patients and providers, and how health literacy strategies create personalized self-management tools and resources for patients [59]. Koh et al. found more health literacy research is needed regarding the creation of accurate, accessible, and actionable health information that is targeted or tailored for specialized populations, such as medically underserved audiences [59].

For U.S.-based researchers, Koh et al. added there are an array of additional health literacy needs and opportunities to evaluate how health literacy interventions and initiatives advance initiatives introduced by the U.S. Affordable Care Act, such as accountable care organizations and patient centered medical homes [59].

Turning to narrower clinical and health care organizational needs, Aldoory, Ryan and Rouhain recently reported a dearth of health literacy research (and evidence) to guide the creation of understandable informed consent forms provided to patients within clinical settings [18]. Aldoory et al. implied the evidence gaps were surprising given the enduring importance of informed consent to patient safety and welfare [18].

Similarly, Rudd recently noted more health literacy research (and evidence) is needed in specific patient-clinical exposure areas such as: unnecessarily dense and complex forms (e.g. hospital entry, consent and discharge forms); clear signage; and enhancing the capacity of visitors to navigate building entrances, passageways, and destination points within hospitals, clinics, and other health care organizations [91]. Rudd noted there is little health literacy research about health care organizational/patient communication practices, such as the creation of a more cheerful, empathetic environment in which health care is delivered in medical offices, hospitals, clinics, and other health care organizations [91].

More broadly, still within health care organizations, Rudd added more health literacy research is needed in areas such as: the clarity of written and spoken clinical communication; assessments of the comparative degree of difficulty of written and posted health information routinely distributed by medical centers; the barriers that discourage people from health information seeking (such as problematic websites, phone interactions, poor maps and signage); and the mismatch between the literacy demands of health materials (written or on-line) and the literacy skills of adults with a secondary school education [91]. 
Rudd also explained more health literacy research is needed within specific areas of patient/provider communication such as: the reading tasks required of patients; understanding patient reading levels; enhancing the clarity of written and spoken communication; removing clinical jargon between health care organizations and culturally diverse audiences; as well as the impact of a health care professional's communication skills on health literacy and patient health outcomes [91]. Rudd added there are evidence gaps in diverse areas such as: the rigorous pilot testing of materials intended for consumers and patients; the measurement and ranking of the communication skills of clinical and health care professionals; and theory driven research based on assessments of consumer and patient interventions [91].

Regarding other scholarly frontiers, the author recently noted the need for research that bridges the conceptual overlaps and gaps among health literacy research and allied fields, such as health communication, and consumer health informatics $[58,66]$. As health information technology evolves and is used to monitor clinical biometrics, inform targeted audiences about health, as well as modify health-related behaviors, there is a pressing need for cross-disciplinary research that embraces consumer health informatics, health communication, and health literacy. There also may be a need to embrace research in related disciplines, such as the public understanding of science.

Overall, this section identifies specific topics and areas where health literacy and health disparities research is needed within diverse areas of clinical care, population health, and health care administration in addition to research that bridges multidisciplinary boundaries. The array of aforementioned topics suggests there are plentiful challenges for future health literacy and health disparities research, which provide opportunities to address existing questions, provide evidence, and furnish new frontiers.

\section{Seeking an expanded, multidimensional conceptual framework of health disparities and health literacy research}

The fourth section, seeking an expanded, multidimensional conceptual approach to health literacy and health disparities research, begins with summaries drawn from the previous subsections and then, provides two examples where some integration has occurred. This is followed by a brief discussion of the similarities between the selected example of health literacy/health disparities expansion and a recent commentary that encourages more integrated research in population health and palliative care.

The previous sections of this chapter strongly suggest an expanded, multidimensional approach to health disparities and health literacy research is desirable partially because the diverse structural and social determinants of health are not mutually exclusive, and probably do not function in isolation. A tenet of the literature that describes structural and social determinants of health is that each of these areas are omnipresent, and each partially helps determine health, or may become part of the everyday experience that occurs when consumers access the health care delivery system.

This strongly suggests that conceptually and operationally, structural and social determinants of health overlap in matters of population and personal health. For example, a conceptual modeling of how demographic differences impact life expectancy probably is not limited to the identified, intervening variables of income and geography within health disparities research. Health disparities findings add there may be array of other sociological, demographic, geographic, and environmental factors that impact life expectancy and health outcomes. In addition, health literacy research suggests health and health care utilization outcomes (but not necessarily life expectancy) may be impacted by an array of underlying factors, such as: chronic misunderstandings of medical terms and instructions among users of the health care delivery system; poor tailoring of health materials to patient or caregiver needs; and the 
anxiety created by an illness and seeking treatment. Similarly, health outcomes (but not necessarily life expectancy) are indirectly impacted by quality of care issues, including: access to care; the quality of clinical research; as well other structural factors. While health disparities, health literacy, and structural challenges within the health care delivery system may or may not predict individual and population health, each may have a role in health's overall determination.

As structural and social determinants are omnipresent, this begs the broader question if they also are interdependent. The latter point is important since if structural and social determinants are interdependent then, research approaches and methods should encompass a range of interactions that traverse structural and social dimensions.

While the IOM health indicators report did not suggest health literacy, health disparities, and structural dimensions are interdependent, the report recommends more inclusive research that integrates structural and social outcome variables [55]. The IOM health indicators report suggests the horizon of future research is to conceive and operationalize population health in terms of more comprehensive approaches [55].

As aforementioned, some evidence that health literacy and health disparities (as social determinants of health and empirical variables) may be interdependent is suggested by the characterization of each dimension as representing intermediate and moderating variables. Since intermediate and moderating variables interact with independent and dependent research measures, this suggests that social and structural dimensions influence health's antecedents and outcomes both in quantitative findings and within research settings. Either way, an underlying research issue that needs to be addressed is the degree of interdependence as well as the interactions among health literacy and health disparities variables within specific settings, such as community based interventions to improve the health and quality of life among vulnerable populations. The uncertainty about the interdependence among social and structural dimensions of health within community interventions or population health initiatives remains a core challenge in the future of health literacy and health disparities research both as individual and possibly collaborative areas of inquiry.

Currently, life skills progression (LSP) research and the NUKA health care approaches by the Southcentral Foundation provide at least two examples of interventions that combine social and structural dimensions as well as suggest future integrative research and practice pathways.

Using Wollensen and Peifer's life skills progression (LSP) conceptual framework, Smith focuses on a secondary analysis of social work, home visitation interventions for a vulnerable population (lower income homes often with single mothers) [94,100]. While LSP research is based on home visitation rather than community-based interventions and the research approach does not use conventional health literacy assessment tools, the LSP findings suggest how expanded conceptual approaches yield insights about the framing of interventions for vulnerable audiences as well as the contributions of structural and social dimensions to health outcomes [94].

For example, the dimensions within the research design in LSP interventions encompass some health disparities, health literacy, structural, as well as other variables. As a dimension, health disparities is assessed by variables such as: housing; food; transportation; insurance; language; education; employment; income; reading level; and self-esteem [29,93,94]. Maternal health literacy is assessed by a composite of surrogate measures such as improvements in: health information seeking; reflective questioning about health; self care; enablement; and empowerment $[29,93,94]$. The structural dimensions of health are assessed by variables such as: use of health services and community resources; preventive practices suggested by a Healthcare Literacy Scale; prenatal care; maternal sick care; and child dental care [29,93,94]. 
Life skills progression research provides four tiers of findings that first, suggest improving maternal health literacy is associated with improvements in parental and child health $[93,94]$. Second, the findings suggest there are significant empirical associations (or interactions) among health disparities, health literacy, and structural variables [100]. Third, the findings suggest health disparities variables vary in their contribution to health outcomes $[29,93,94]$. Interestingly, immigration status, reading levels, and less than 12th grade education are less associated with outcomes than other demographic measures. Fourth, the findings suggest structural, health literacy, and health disparities dimensions may be interdependent, or statistically converge to improve family health outcomes $[29,93,94]$.

Overall, the LSP findings demonstrate how a framework can be developed to assess some of the interactions across the social and structural dimensions that are discussed within this chapter.

While LSP focuses on interventions in non-clinical settings, the Southcentral Foundation's NUKA system of care integrates health's social, structural, and cultural dimensions within a medical center's routine health delivery to vulnerable populations (Alaska Natives) [46,47]. NUKA (a Native Alaskan word for 'giant structures and living things') tries to address health care's 'three aims' (improve patient experiences, health, and restrain costs) via screening for health's social, structural, and cultural dimensions and responding to operant individual and family needs [46].

The NUKA approach to integrated care is more than incorporating physical and mental health interdisciplinary teams within patient care. The NUKA approach includes routine health interventions that address structural health dimensions such as: significantly reducing patient waiting times; improving immediate access to clinicians and other specialists; creating innovative relationships among patients and providers; as well as rethinking how to listen to patients [46,47]. It includes routine interventions that address health disparities such as: strategies designed to revitalize treatments for mental and emotional health; and projects designed to build a sense of community heritage and pride [46,47]. NUKA includes routine interventions that address health literacy such as: learning circles that attempt to significantly boost patient engagement; education about specific issues such as spousal and child abuse, alcoholism, and obesity; and encouraging providers to help patients understand their diagnoses or condition $[46,47]$. NUKA also includes routine interventions that address individual and community health needs as well as conventional patient care $[46,47]$.

Essentially, the NUKA approach to integrated care partially incorporates health disparities, health literacy, behavioral health, immediate clinical needs, prevention, wellness, and cost savings interventions simultaneously, depending on individual, family, or neighborhood requirements. As a result, the NUKA approach additionally provides a clinically based organizational template for some of the expanded research models and intervention assessments that are suggested within this chapter.

Moreover, the NUKA approach to care suggests a busy medical center and health care delivery system can successfully frame care in terms of building relations that seek to improve a patient's quality of life and personal development across their life course, foster community development, as well as treat a patient's disease or condition. Similarly, the LSP interventions are grounded in efforts to improve a client's quality of life as well as their personal development as a foundation to enhance the treatment of specific patient clinical needs.

In both the LSP and NUKA examples, the conceptual framing of individual and population health reflects 'a more comprehensive and holistic picture of life,' as recently discussed by Casarett and Teno [31]. Casarett and Teno add that a more comprehensive and holistic concept of life also fosters a foundation for the increased integration of population health and palliative care research and practice [31]. Hence, the links between the integration of health's social and structural dimensions that are identified in this chapter may parallel suggestions to integrate other population health fields. This additionally 
suggests an integrated conceptual framework is attracting interest as a strategy to enhance the future of research and practice within health care.

\section{Conclusion}

At present, there are foundational areas of clinical, public health, and health administration with evidence gaps and where health disparities and health literacy research has yet to occur, basic research needs to be conducted, and inconsistent results suggest a need for further research $[17,18,26,69,85]$. As research constructs, health disparities and health literacy also are currently best described as intermediate, moderating research variables that are associated with therapeutic individual, social, cultural, clinical, and health care organizational outcomes.

As aforementioned, the horizon for research is to integrate health's social and structural dimensions more holistically and assess the degree social and structural dimensions may or may not be interdependent in influencing health outcomes and care utilization. The NUKA and LSP examples additionally suggest social and structural dimensions can be part of a broader effort to nurture health and well being as well as integrate clinical care with services that often are currently seen as provided by social work, public health initiatives, educational development, public works, and other areas that impact human development.

Overall for health disparities and health literacy researchers, this is both a daunting and exciting time to enter or be a part of fields where vital issues and questions are irresolute and the potential for imaginative research and practice is multidimensional and expansive. The future challenge of health disparities and health literacy research is to frame and assess interventions in diverse communities and provide more tailored, theory-based, and constructive interventions. At the same time, the chapter suggests the future challenge of health disparities and health literacy research is to advance as well as reinvent both fields via collaborative efforts and more integrated theoretical foundations, constructs, and operations.

Finally, a future opportunity for health disparities and health literacy researchers is the creation of international academic organizations that seek to foster collaborative research and practice. Currently, both health disparities and health disparities (as arenas of research and practice) need a member-based, international, academic infrastructure to host periodic meetings, publish refereed journals, provide news about research and practice to members, encourage practitioner-scholar, practitioner-practitioner, scholar-scholar dialogue, establish leadership, provide a clearinghouse for practitioner services, such as the Institute for Healthcare Advancement's health literacy listserv, and organize both fields into a more coherent whole [10].

While some of these needs may be served by existing international conferences and organizations, progress might be accelerated by the development of a focal point for interaction and diffusion among the world's health disparities and literacy researchers and practitioners. In addition, the lack of a central organization to define health literacy and health disparities research collaboration and professionalism lets the fields' future be defined externally - by clinical medicine, public health, and other disciplines its scholars and practitioners represent. Certainly, the development of an international health disparities and literacy disciplinary infrastructure to elevate research and practice standards (similar to professional societies in clinical, medical administration, public health, and social science disciplines) remains an area where future researchers can contribute to the evolution, growth, gravitas, and success of both fields. 


\section{References}

[1] http://www.healthnewsreview.org/2016/05/superficial-coverage-of-medical-errors-could-leave-erroneous-impressionwith-readers/, Retrieved September 1, 2016.

[2] http://www.centreforliteracy.qc.ca/health_literacy/calgary_charter. Retrieved August 4, 2016.

[3] http://www.cdc.gov/minorityhealth/chdireport.html, Retrieved September 2, 2016.

[4] http://healthjournalism.org/core-topic.php?id=6\&page=glossary\#HealthEquity, Retrieved May 15, 2016.

[5] http://www.rwjf.org/en/about-rwjf/newsroom/features-and-articles/Commission.html, Retrieved August 1, 2016.

[6] http://governor.alabama.gov/newsroom/2016/04/executive-order-number-18-2/, Retrieved June 20, 2016.

[7] http://nces.ed.gov/naal/health_results.asp, Retrieved May 16, 2016.

[8] http://www.ncqa.org/Portals/0/Publications/Resource\%20Library/NCQA_Primer_web.pdf, Retrieved May 11, 2016.

[9] http://nnlm.gov/outreach/consumer/hlthlit.html, Retrieved June 15, 2016.

[10] http://listserv.ihahealthliteracy.org/scripts/wa.exe?INDEX, Retrieved April 23, 2016.

[11] https://www.nih.gov/precision-medicine-initiative-cohort-program/scale-scope, Retrieved August 20, 2016.

[12] https://kaiserfamilyfoundation.files.wordpress.com/2013/01/8396.pdf, Retrieved September 2, 2016.

[13] https://chirr.nlm.nih.gov, Retrieved September 8, 2016.

[14] https://www.nlm.nih.gov/services/queries/health_literacy.html, Retrieved July 11, 2016.

[15] M.A. Abrams, P. Klass and B.P. Dreyer, Health literacy and children: Recommendations for action, Pediatrics 124 (2009), S327-S331. doi:10.1542/peds.2009-1162I.

[16] N.E. Adler, Overview of health disparities, in: Examining the Health Disparities Research Plan of the National Institutes of Health, Institute of Medicine (US) Committee on the Review and Assessment of the NIH's Strategic Research Plan and Budget to Reduce and Ultimately Eliminate Health Disparities, G.E. Thomson, F. Mitchell and M.B. Williams, eds, Unfinished Business, Washington, 2006, National Academies Press.

[17] Agency for Healthcare Research and Quality, Health literacy interventions and outcomes: An updated systematic review, Rockville, MD, Agency for Healthcare Research and Quality, Evidence report/technology assessment Number 199, 2011, http://www.ahrq.gov/downloads/pub/evidence/pdf/literacy/literacyup.pdf.

[18] L. Aldoory, K.E.B. Ryan and A.M. Rouhain, Best practices and new models of health literacy for informed consent: Review of the impact of informed consent regulations on health literate communications, in: Informed Consent and Health Literacy: A Workshop, Institute of Medicine of the National Academies Health Literacy Roundtable, Washington, 2014.

[19] S.V. Altin, I. Finke, S. Kautz-Freimuth and S. Stock, The evolution of health literacy assessment tools: A systematic review, BMC Public Health 14 (2014), 1207. doi:10.1186/1471-2458-14-1207.

[20] L.M. Anderson, K.L. Adeney, C. Shinn, S. Safranek, J. Buckner-Brown and L.K. Krause, Community coalition-driven interventions to reduce health disparities among racial and ethnic minority populations, Cochrane Database of Systematic Reviews 6 (2015), CD009905. doi:10.1002/14651858.CD009905.pub2.

[21] S.C. Bailey, C.U. Oramasion and M.S. Wolf, Rethinking adherence: A health literacy-informed model of medication self-management, J. Health Commun. 18(Suppl. 1) (2013), 20-30. doi:10.1080/10810730.2013.825672.

[22] D.W. Baker, The associations between health literacy and health outcomes: Self-reported health, hospitalization, and mortality, in: Office of the Surgeon General, Proceedings of the Surgeon General's Workshop on Improving Health Literacy, Office of the Surgeon General (US), Rockville, MD, 2006, http://www.ncbi.nlm.nih.gov/books/NBK44260/\# proc-healthlit.panel1.s14, Retrieved May 2, 2016.

[23] M. Baker, 1500 scientists lift the lid on reproducibility, Nature 533(7604) (2016), 452-454. doi:10.1038/533452a.

[24] C. Bambra, Health Divides: Where You Live Can Kill You, Policy Press, Bristol, UK, 2016.

[25] D.A. Barr, Health Disparities in the United States: Social Class, Race, Ethnicity \& Health, 2nd edn, Johns Hopkins University Press, Baltimore, 2014.

[26] N.D. Berkman, S.L. Sheridan, K.E. Donahue, D.J. Halpern, A. Viera, K. Crotty, A. Holland, M. Brasure, K.N. Lohr, E. Harden, E. Tant, I. Wallace and M. Viswanathan, Health literacy interventions and outcomes: An updated systematic review. Evidence Report/Technology Assessment No. 199, RTI International-University of North Carolina Evidencebased Practice Center under contract No. 290-2007-10056-I, AHRQ Publication Number 11-E006, Rockville, MD. Agency for Healthcare Research and Quality, 2011.

[27] D.M. Berwick, A user's manual for the IOM 'quality chasm' report, Health Aff. 21(3) (2002), 80-90. doi:10.1377/ hlthaff.21.3.80.

[28] P. Braveman and S. Gruskin, Defining equity in health, J. Epidemiol. Community Health 57(4) (2003), $254-258$. doi:10.1136/jech.57.4.254.

[29] L.N. Carroll, S.A. Smith and N.R. Thomson, Parents as teachers health literacy demonstration project: Integrating an empowerment model of health literacy promotion into home-based parent education, Health Promot. Pract. 16(2) (2015), 282-290. doi:10.1177/1524839914538968.

[30] O. Carter-Pokras and C. Baquet, Viewpoint: What is a 'health disparity'?, Public Health Reports 117 (2002), $426-434$. 
[31] D. Casarett and J. Teno, Why population health and palliative care need each other, JAMA 316(1) (2016), 27-28. doi:10.1001/jama.2016.5961.

[32] R. Chetty, M. Stepner, S. Abraham, S. Lin, B. Scudent, N. Tuner, A. Bergeron and D. Cutler, The association between income and life expectancy in the United States, 2001-2014, JAMA 315(16) (2016), 1750-1766. doi:10.1001/ jama.2016.4226.

[33] F.S. Collins and H. Varmus, A new initiative on precision medicine, N. Engl. J. Medicine 372(9) (2015), 793-795. doi:10.1056/NEJMp1500523.

[34] A. Coulter, Engaging Patients in Healthcare, Open University Press, Berkshire, England, 2011.

[35] J. Currie and H. Schwandt, Inequality in mortality decreased among the young while increasing for older adults, 19902010, Science 352(6286) (2016), 708-712. doi:10.1126/science.aaf1437.

[36] P. Demoly, G. Passalacqua, O. Pfaar, J. Sastre and U. Wahn, Patient engagement and patient support programs in allergy immunotherapy: A call to action for improving long-term adherence, Allergy Asthma Clin. Immunol. 29(12) (2016), 34. doi:10.1186/s13223-016-0140-2.

[37] D.A. Dewalt and A. Hink, Health literacy and child health outcomes: A systematic review of the literature, Pediatrics 124(Suppl. 3) (2009), S265-S274. doi:10.1542/peds.2009-1162B.

[38] C.C. Doak, L.G. Doak, G.H. Friedell and C.D. Meade, Improving communication for cancer patients with low literacy skills: Strategies for clinicians, CA Cancer J. Clin. 48(3) (1998), 151-162. doi:10.3322/canjclin.48.3.151.

[39] C.C. Doak, L.G. Doak and J.H. Root, Teaching Patients with Low-Literacy Skills, 2nd edn, JB Lippincott, Philadelphia, 1996.

[40] L.G. Doak and C.C. Doak, Lowering the silent barriers for patients with low literacy skills, Promot. Health. 8(4) (1987), 6-8.

[41] L.G. Doak, C.C. Doak and C.D. Meade, Strategies to improve cancer education materials, Oncol. Nurs. Forum. 23(8) (1996), 1305-1312.

[42] L.P. Freedman and J. Inglese, The increasing urgency for standards in basic biological research, Cancer Res. 74(15) (2014), 4024-4029. doi:10.1158/0008-5472.CAN-14-0925.

[43] A. Gawande, Complications: A Surgeon's Notes on an Imperfect Science, Metropolitan Books, New York, 2002.

[44] A. Gawande, The cost conundrum; what a Texas town can teach us about health care, 2009, http://www.newyorker.com/ magazine/2009/06/01/the-cost-conundrum, Retrieved September 2, 2016.

[45] C.E. Gessert, I.V. Haller and B.P. Johnson, Regional variation in care at the end of life: Discontinuation of dialysis, $B M C$ Geriatr. 13 (2013), 39. doi:10.1186/1471-2318-13-39.

[46] K. Gottlieb, The Nuka system of care: Improving health through ownership and relationships, Int. J. Circumpolar. Health 5 (2013), 72. doi:10.3402/ijch.v72i0.21118.

[47] K. Gottlieb, I. Sylvester and D. Eby, Transforming your practice: What matters most, Fam. Pract. Manag. 15(1) (2008), $32-38$.

[48] J. Gregory and S. Miller, Science in Public: Communication, Culture \& Credibility. Perseus, New York, 2000.

[49] G. Halvorson, Ending racial, ethnic, and cultural disparities, in American Health Care, CreateSpace Independent Publishing Platform, N. Charleston, 2013.

[50] D.M. Hartung, D.A. Zarin, J.M. Guise, M. McDonaugh, R. Paynter and M. Helfand, Reporting discrepancies between the ClinicalTrials.gov database and peer-reviewed publications, Ann. Intern. Med. 160(7) (2014), 477-483. doi:10.7326/M13-0480.

[51] J.N. Haun, M.A. Valerio, L.A. McCormack, K. Sorensen and M.K. Paasche-Orlow, Health literacy measurements: An inventory and descriptive summary of 51 instruments, J. Health Commun. 10(Suppl. 2) (2014), 302-333. doi:10.1080/ 10810730.2014.936571.

[52] J.H. Hibbard, E.R. Mahoney, J. Stockard and M. Tusler, Development and testing of a short form of the patient activation measure, Health Serv. Res. 40(6 Pt. 1) (2005), 1918-1930. doi:10.1111/j.1475-6773.2005.00438.x.

[53] Institute of Medicine of the National Academies, Crossing the quality chasm: A new health system for the 21st century, The National Academies Press, Washington, 2001.

[54] Institute of Medicine of the National Academies, Health literacy: A prescription to end confusion, The National Academies Press, Washington, 2004.

[55] Institute of Medicine of the National Academies. Committee on the state of the USA health indicators. Board on population health and public health practice, The National Academies Press, Washington, 2009.

[56] Institute of Medicine of the National Academies, Health literacy: improving, health, health systems, and health policy around the world, The National Academies Press, Washington, 2013.

[57] Institute of Medicine of the National Academies, Informed consent and health literacy: A workshop, Institute of Medicine Health Literacy Roundtable, Washington, DC, 2014.

[58] A. Keselman, R.A. Logan, C.A. Smith, G. LeRoy and Q. Zeng-Treitler, Developing informatics tools and strategies for consumer-centered health communication, J. Am. Med. Inform. Assoc. 15(4) (2008), 475-483. doi:10.1197/ jamia.M2744. 
[59] H.K. Koh, C. Baur, C. Brach, L.M. Harris and J.N. Rowden, Towards a systems approach to health literacy research, J. Health Commun. 18(1) (2013), 1-5. doi:10.1080/10810730.2013.759029.

[60] H.K. Koh, D.M. Berwick, C.M. Clancy, C. Baur, C. Brach, L.M. Harris and E.G. Zerhusen, New federal policy initiatives to boost health literacy can help the nation move beyond the cycle of costly 'crisis care', Health Aff. 31(2) (2012), 434443. doi:10.1377/hlthaff.2011.1169.

[61] L.T. Kohn, J.M. Corrigan and M.S. Donaldson, eds, To Err Is Human: Building a Safer Health System, The National Academies Press, Washington, 2000.

[62] G.L. Kreps and L. Neuhauser, New directions in eHealth communications: Opportunities and challenges, Patient Edu. Couns. 78 (2010), 329-336. doi:10.1016/j.pec.2010.01.013.

[63] M. Kutner, E. Greenberg, Y. Jin and C. Paulsen, The Health Literacy of America's Adults: Results from the 2003 National Assessment of Adult Literacy (NCES2006-483). U.S. Department of Education, National Center for Education Statistics, Washington, 2006.

[64] B. Lindstrom and E.M. Salutogenesis, J. Epidemiol. Community Health 59(6) (2005), 440-442. doi:10.1136/ jech.2005.034777.

[65] R.A. Logan, Health campaigns research, in: Routledge Handbook of Public Communication of Science and Technology, M. Bucci and B. Trench, eds, 2nd edn, Routledge, New York, 2014, pp. 198-213.

[66] R.A. Logan, Health literacy research, in: Meeting Health Information Needs Outside of Healthcare: Opportunities and Challenges, C. Arnott-Smith and A. Keselman, eds, Chandos, Waltham, MA, 2015, pp. 19-38. doi:10.1016/B978-008-100248-3.00002-0.

[67] R.A. Logan, W.F. Wong, M. Villaire, G. Daus, T.A. Parnell, E. Willis and M.K. Paasche-Orlow, Health literacy: A necessary element for achieving health equity, Discussion Paper, National Academy of Medicine, Washington, DC, 2015, http://www.nam.edu/perspectives/2015/Health-literacy-a-necessary-element-for-achieving-health-equity.

[68] M.A. Makary and M. Daniel, Medical error-the third leading cause of death in the US, BMJ 353 (2016), i2139. doi:10.1136/bmj.i2139.

[69] L. McCormack, J. Haun, K. Sorensen and M. Valerio, Recommendations for advancing health literacy measurement, J. Health Commun. 18(Suppl. 1) (2013), 9-14. doi:10.1080/10810730.2013.829892.

[70] J.D. Miller, Public understanding of, and attitudes towards, scientific research: What we know and what we need to know, Public Underst. Sci. 13(2) (2004), 273-294. doi:10.1177/0963662504044908.

[71] J.J. Mitchell Jr., The findings of the Dartmouth Atlas Project: A challenge to clinical and ethical excellence in end-of-life care, J. Clin. Ethics Fall. 22(3) (2011), 267-276.

[72] T. Nakaya and D. Dorling, Geographical inequalities of mortality by income in two developed island countries: A crossnational comparison, Soc. Sci. Med. 60(12) (2005), 2865-2875. doi:10.1016/j.socscimed.2004.11.007.

[73] National Academies of Sciences Engineering Medicine, Precision medicine and health literacy: A workshop, National Institute of Medicine Roundtable on Health Literacy, Washington, 2016.

[74] D. Nelkin, Selling Science: How the Press Covers Science and Technology, W.H. Freeman, New York, 1995.

[75] L. Neuhauser and G.L. Kreps, Rethinking communication in the E-health era, J. Health Psychol. 8(1) (2003), 7-22. doi:10.1177/1359105303008001426.

[76] O.K. Nguyen, E.A. Halm and A.N. Makam, Relationship between hospital financial performance and public reported outcomes, J. Hosp. Med. 11(7) (2016), 481-488. doi:10.1002/jhm.2570.

[77] D. Nutbeam, Health literacy as a public health goal: A challenge for contemporary health education and communication strategies into the 21st century, Health Promotion Int. 15 (2000), 259-267. doi:10.1093/heapro/15.3.259.

[78] D. Nutbeam, The evolving concept of health literacy, Soc. Sci. Med. 67 (2008), 2072-2078. doi:10.1016/ j. socscimed.2008.09.050.

[79] A.M. O'Hare, R.A. Rodriguez, S.M. Halipern, E.B. Larson and T.M. Kurella, Regional variation in health care intensity and treatment practices for end-stage renal disease in older adults, JAMA 304(2) (2010), 180-186. doi:10.1001/ jama.2010.924.

[80] B. O'Neill, D. Goncalves, I. Ricci-Cabello and S. Ziebland, An overview of self-administered health literacy instruments, PLOS One 9(12) (2014), e109110. doi:10.1371/journal.pone.0109110.

[81] M.K. Paasche-Orlow and M.S. Wolf, Promoting health literacy research to reduce disparities, J. Health Commun. 2010(15 Suppl. 2) (2010), 34-41. doi:10.1080/10810730.2010.499994.

[82] R.M. Parker and S.C. Ratzen, Health literacy: A second decade of distinctions for Americans, J. Health Commun. 15(Suppl. 2) (2010), 20-33. doi:10.1080/10810730.2010.501094.

[83] E.A. Pascoe and L.S. Richman, Perceived discrimination and health: A meta-analytic, Review Psychol. Bul. 135(4) (2009), 531-554. doi:10.1037/a0016059.

[84] A. Penman-Aguilar, M. Talih, D. Huang, R. Moonesinghe, K. Bouye and G. Beckles, Measurement of health disparities, health inequities, and social determinants of health to support the advancement of health equity, J. Public Health Manag. Pract. 22(Suppl. 1) (2016), S33-S42. doi:10.1097/PHH.0000000000000373. 
[85] A. Pleasant, Advancing health literacy measurement: A pathway to better health and health system performance, J. Health Commun. 19(12) (2014), 1481-1496. doi:10.1080/10810730.2014.954083.

[86] A. Pleasant, Health literacy measurement. Lecture 3 of 5 better health: evaluating health communication symposium, National Library of Medicine, Bethesda (MD), 2013, http://videocast.nih.gov. Retrieved June 16, 2016.

[87] A. Pleasant, J. McKinney and R.V. Rikard, Health literacy measurement: A proposed research agenda, J Health Commun. 16(Suppl. 3) (2011), 11-21. doi:10.1080/10810730.2011.604392.

[88] A. Pleasant, R.E. Rudd, C. O’Leary, M.K. Paasche-Orlow, M.P. Allen, W. Alvarado-Little, L. Myers, K. Parson and S. Rosen, Considerations for a New Definition of Health Literacy, National Academy of Medicine, Washington, 2016, https://nam.edu/considerations-for-a-new-definition-of-health-literacy/.

[89] S.C. Ratzan, The future of health communication: Innovating through partnerships, Metode Science Studies Journal (2015). doi:10.7203/metode.6.7096.

[90] Royal College of General Practitioners, Health Literacy: Report from an RCGP-Led Health Literacy Workshop, Royal College of General Practitioners, London, UK, 2014.

[91] R.E. Rudd, Needed action in health literacy, Journal Health Psychol. (2013). doi:10.1177/1359105312470128.

[92] M.H. Shah, Prior authorization: Undermining our health care system, J. Med. Assoc. Ga. 104(2) (2015), 3.

[93] S.A. Smith, Health literacy and social service delivery, in: New Directions in Behavioral Health: Service Delivery Strategies for Vulnerable Populations, S.A. Estrine, H.G. Arthur, R.T. Hettenbach and M.G. Messina, eds, Springer Publishing, New York, 2011.

[94] S.A. Smith and E.J. Moore, Health literacy and depression in the context of home visitation, Matern. Child Health J. 16(7) (2012), 1500-1508. doi:10.1007/s10995-011-0920-8.

[95] K. Sorensen, S. Van den Broucke, J. Fullam, G. Doyle, J. Pelikan, Z. Slonska, H. Brand and HLS-EU Consortium Health Literacy Project European, Health literacy and public health: A systematic review and integration of definitions and models, BMC Public Health 12(80) (2012). doi:10.1186/1471-2458/12/80.

[96] T.C. Tsai, K.E. Joynt, E.J. Orav, A.A. Gawande and A.K. Jha, Variation in surgical readmission rates and quality of hospital care, N. Engl. J. Med. 369(12) (2013), 1134-1142. doi:10.1056/NEJMsa1303118.

[97] J.M. Unger, E. Cook, E. Tai and A. Bleyer, The role of clinical trial participation in cancer research: Barriers, evidence, and strategies, Am. Soc. Clin. Oncol. Educ. Book 35 (2015), 185-198. doi:10.14694/EDBK_156686.

[98] W.B. Weeks and J.N. Weinstein, Unraveled: Prescriptions to repair a broken health system, CreateSpace Independent Publishing Platform, N. Charlestown, 2016.

[99] S. White, Assessing the Nation's Health Literacy: Key Concepts and Findings of the National Assessment of Adult Literacy (NAAL), American Medical Association Foundation, Chicago, 2008.

[100] L. Wollesen and K. Peifer, Life Skills Progression: An Outcome and Intervention Planning Instrument for Use with Families at Risk, Brookes, Baltimore, MD, 2006.

[101] S.H. Woolf and J.Q. Purnell, The good life: Working together to promote opportunity and improve population health and well-being, JAMA 315(16) (2016), 1706-1708. doi:10.1001/jama.2016.4263. 